\title{
Incremental increases in economic burden parallels cardiometabolic risk factors in the US
}

\section{R Brett McQueen' \\ Vahram Ghushchyan ${ }^{1,2}$ \\ Temitope Olufade ${ }^{3}$ \\ John J Sheehan ${ }^{4}$ \\ Kavita $\bigvee$ Nair $^{\prime}$ \\ Joseph J Saseen ${ }^{1,5}$}

'Department of Clinical Pharmacy, University of Colorado Skaggs School of Pharmacy and Pharmaceutical Sciences, Anschutz Medical Campus, Aurora, CO, USA; ${ }^{2}$ College of Business and Economics, American University of Armenia, Yerevan, Armenia; ${ }^{3}$ AstraZeneca, Wilmington, $\mathrm{DE},{ }^{4}$ AstraZeneca, Fort Washington, PA, ${ }^{5}$ Department of Family Medicine, University of Colorado Anschutz Medical Campus, Aurora, CO, USA

Correspondence: Joseph J Saseen Department of Clinical Pharmacy, University of Colorado Skaggs School of Pharmacy and Pharmaceutical Sciences, Mail Stop C238, 12850 E Montview Boulevard, Aurora, CO 80045, USA Tel +I 3037242656

Email joseph.saseen@ucdenver.edu
This article was published in the following Dove Press journal: Diabetes, Metabolic Syndrome and Obesity:Targets and Therapy 2 August 2016

Number of times this article has been viewed

Objective: Estimate the economic burden associated with incremental increases in the number of cardiometabolic risk factors (CMRFs) in the US.

Methods: We used the nationally representative Medical Expenditure Panel Survey from 2010 to 2012 to create a retrospective cohort of people based on the number of CMRFs (one, two, and three or four), and a comparison cohort of people with zero CMRFs. CMRFs included abdominal obesity, elevated blood pressure, elevated triglycerides, and elevated glucose and were defined using diagnostic codes, prescribed medications, and survey responses. Adjusted regression analysis was developed to compare health expenditures, utilization, and lost-productivity differences between the cohorts. Generalized linear regression was used for health care expenditures, and negative binomial regression was used for utilization and productivity, controlling for individual characteristics.

Results: The number of CMRFs was associated with significantly more annual utilization, health care expenditures, and reduced productivity. As compared with people with zero CMRFs, people with one, two, and three or four CMRFs had 1.15 (95\% confidence interval [CI]: 1.06, 1.24), 1.37 (95\% CI: 1.25, 1.51), and 1.39 (95\% CI: 1.22, 1.57) times higher expected rate of emergency room visits, respectively. Compared with people with zero CMRFs, people with one, two, and three or four CMRFs had increased incremental health care expenditures of US\$417 (95\% CI: \$70, \$763), US\$2,326 (95\% CI: \$1,864, \$2,788), and US\$4,117 (95\% CI: \$3,428, $\$ 4,807)$, respectively. Those with three or four CMRFs reported employment of $60 \%$, compared with $80 \%$ in patients with zero CMRFs. People with three or four CMFRs had 1.75 (95\% CI: $1.42,2.17)$ times higher expected rate of days missed at work due to illness, compared with people with zero CMRFs.

Conclusion: Our findings demonstrate a direct association between economic burden and number of CMRFs. Although this was expected, the increase in burden that was independent from the cost of cardiovascular disease was surprising.

Keywords: economic burden, cardiometabolic risk factors, cardiovascular disease

\section{Introduction}

Cardiometabolic risk factors (CMRFs) such as elevated fasting glucose, elevated blood pressure, low high-density lipoprotein cholesterol, elevated fasting triglycerides, and abdominal obesity often coexist in the same individual. The presence of multiple CMRFs has been broadly termed as the "metabolic syndrome". ${ }^{1}$ Although definitions of "metabolic syndrome" from different organizations vary regarding the exact number of criteria and cut points for each parameter, the presence of these risk factors often results in increased risk of cardiovascular $(\mathrm{CV})$ disease-related morbidity and 
mortality. ${ }^{2-7} \mathrm{CV}$ disease still poses a significant burden in the US and accounted for one in three deaths and annual costs for treating CV disease were $\sim$ US\$320.1 billion in $2011 .^{8}$

Health interventions in the US have reduced the prevalence of elevated fasting triglycerides and elevated blood pressure from 1999 to $2010 .{ }^{8}$ However, prevalence of elevated glucose and elevated waist circumference has continued to rise over this same time period, particularly among children and adolescent populations. Age-adjusted national US prevalence of metabolic syndrome has decreased for women but prevalence remains high for men. Given the increasing prevalence of elevated glucose and waist circumference among children and adolescents, and an aging population, the prevalence and economic burden of CMRFs will continue to rise.

Prior to the onset of $\mathrm{CV}$ disease, concomitant CMRFs are associated with a significant increase in medical expenditures and lost productivity. ${ }^{9-12}$ Several studies suggest that a higher number of CMRFs and specific combinations of CMRFs (eg, diabetes and obesity) are associated with significant CV-related events and costs. ${ }^{13-16}$ However, there is a lack of evidence reporting both direct medical costs and lost productivity associated with incremental increases in CMRFs regardless of concomitant conditions. The primary objective of this study was to estimate the economic burden as a function of direct medical costs and lost productivity associated with incremental increases in the number of CMRFs using recently available nationally representative data from the US noninstitutionalized population.

\section{Methodology}

\section{Data source and study population}

We utilized the nationally representative, publicly available panel database, the Medical Expenditure Panel Survey (MEPS). The MEPS tracks individual and household healthrelated, demographic, and socioeconomic characteristics. ${ }^{17}$ To provide estimates that are representative of a national US population, the Household Component of the MEPS (MEPSHC) panels have oversampled subgroups of individuals such as African-Americans, Hispanics, Asians, low-income households, and those likely to incur high medical expenditures. At the time of the analysis, the most recent available file was for 2012. We combined the 2010-2012 MEPS full-year consolidated, medical, and pharmacy utilization data files to generate an analytical cohort with robust sample size. The Colorado Multiple Institutional Review Board considers this research to be exempt from requiring board approval because MEPS is a publicly available data source.

\section{Population of inference}

The study cohort consisted of noninstitutionalized adult US individuals (18 years of age and above) with at least one and up to four CMRFs. The study cohort was defined using a combination of International Classification of Diseases, ninth revision (ICD-9) codes, National Drug Codes, and survey responses. We calculated the number of CMRFs for each patient and stratified the population into four mutually exclusive and exhaustive groups: those with zero (comparison group), one, two, and a group with three or four CMRFs.

\section{Measures}

\section{Definition of CMRFs}

The following CMRFs were used to calculate the number of CMRFs:

1. Abdominal obesity: Defined as body mass index (BMI) $\geq 27 \mathrm{~kg} / \mathrm{m}^{2}$. Previous research has suggested treatment of overweight individuals when other CMRFs are present should begin at a $\mathrm{BMI} \geq 27 \mathrm{~kg} / \mathrm{m}^{2} .{ }^{18}$

2. Elevated blood pressure: Defined as having at least one ICD-9 code for hypertension (401.x) and at least one prescription for an antihypertensive agent (angiotensinconverting enzyme inhibitor, angiotensin receptor blocker, beta blocker, calcium channel blocker, diuretic, direct renin inhibitor, alpha blocker, alpha agonist, aldosterone antagonist, or direct arterial vasodilator) during a survey year.

3. Elevated triglycerides: Defined as having at least one prescription for a triglyceride-lowering medication (fibrate, niacin, or omega-3 fatty acids) during a survey year.

4. Elevated glucose: Defined as having at least one ICD-9 code for diabetes (250.x) and at least one prescription for an antihyperglycemic medication (biguanide, sulfonylurea, thiazolidinedione, insulin, alpha-glucosidase inhibitor, incretin mimetic, or dipeptidyl peptidase-4 inhibitor) during a survey year.

\section{Other key independent variables}

Two types of independent variables were included in the analysis: clinical and demographic. Demographic variables available in MEPS include sex (male, female), age in years (18-34, 35-49, 50-64, 65-79, 80+), race (white, black, American Indian, and other race), ethnicity (Hispanic/ non-Hispanic), health insurance type (private, public, dual covered, and uninsured), highest degree completed (no degree, less than bachelors, bachelors, masters, and above), family income (classified using percentage of federal poverty level), and region of the country (Northeast, Midwest, South, 
West). Clinical variables included other chronic medical conditions coded as numerical counts of conditions other than the four CMRFs described earlier. The numerical count of other conditions was used as an adjustment variable in the regression analyses.

\section{Outcome measures}

Outcome measures in this study were health care utilization, expenditures, and lost productivity. Health care utilization included the number of outpatient visits, number of emergency room visits, number of inpatient visits, inpatient length of stay, and number of prescriptions including refills. All-cause health care expenditures in MEPS consist of direct payments for all health care utilization during the calendar year, including out-of-pocket payments and payments made by private and public insurance. Health care expenditures were estimated as total health care expenditures (pharmacy + medical). All expenditures were estimated in 2014 US dollars using the medical care component of Consumer Price Index. Two measures of workplace productivity were estimated: number of days missed at work and employed or not employed during (including part-time employment status) the entire year.

\section{Statistical analyses}

Descriptive statistics were estimated for utilization, expenditures, lost productivity, and other clinical and demographic variables. Regression models for health care utilization, expenditures, and lost productivity reported incremental differences for those with one, two, three, or four of the CMRFs defined in this study compared with the general population with zero CMRFs. Regression models adjusted for all relevant clinical and demographic variables, including age, sex, race, ethnicity, region, education, number of other chronic conditions, family income, and insurance status. Negative binomial models were used for the adjusted health care utilization analysis and the lost-productivity analysis for the number of missed days at work. Incidence rate ratios were reported for utilization-related count variables. Health care expenditures often contain a large percentage of zero costs and are heavily right-skewed. To account for these statistical issues, we used generalized linear modeling with log link and gamma family distribution for health care expenditures. Adjusted differences in health care expenditure outcomes were reported as marginal effects $(\mathrm{d} y / \mathrm{d} x)$ as compared with those with zero CMRFs. A logistic regression model was developed to estimate the adjusted difference in employment between groups and reported as odds ratios. All analyses were conducted using Stata ${ }^{\circledR}$ version 12 (StataCorp LP, College Station, TX, USA) incorporating MEPS survey design variables (eg, probability weights).

\section{Sensitivity analyses}

We performed a sensitivity analysis by splitting the three or four CMRFs group into three and four CMRFs separately. We ran all unadjusted and adjusted analyses with three and four CMRFs separated.

\section{Results}

There were 43,037 people with at least one CMRF; 30,849 had zero CMRFs; 29,647 had one CMRF; 9,392 had two CMRFs; and 3,998 had three or four CMRFs (Table 1). Among the same sample, the most prevalent CMRF was abdominal obesity $(n=36,638)$ followed by elevated blood pressure $(n=16,830)$ (Table 2$)$. Increasing number of CMRFs was most prevalent among lower educated, older males. Those with a higher number of CMRFs had a higher number of other chronic conditions (ie, excluding CMRFs) as compared with the average MEPS population. The number of other chronic conditions was highest for people with elevated triglycerides (3.05), elevated glucose (2.61), and elevated blood pressure (2.54) as compared with the average MEPS population (1.35).

Significant increases in unadjusted and adjusted utilization and expenditures were observed as the number of CMRFs increased (Table 3 and Figure 1). As compared with people with zero CMRFs, people with one, two, and three or four CMRFs had 1.15 (95\% CI: 1.06, 1.24), 1.37 (95\% CI: 1.25, $1.51)$, and 1.39 (95\% CI: 1.22, 1.57) times higher expected rate of emergency room visits, respectively, after adjusting for clinical and demographic characteristics. Overall adjusted annual health care expenditures were approximately double for those with three or four CMRFs at US\$7,640 compared with US\$3,523 and US\$3,940 for those with zero CMRFs and one CMRF, respectively (Figure 1).

People with three or four CMRFs reported employment of $60 \%$ compared with $80 \%$ in people with zero CMRFs (Table 4). After adjusting for clinical and demographic characteristics, on average people with three or four CMRFs had 1.38 (95\% CI: $1.17,1.63)$ times higher odds of reporting not being employed as compared with those with zero CMRFs. After adjusting for clinical and demographic characteristics, people with one, two, and three or four CMRFs had 1.21 (95\% CI: 1.09, 1.34), 1.55 (95\% CI: 1.31, 1.82), and 1.75 (95\% CI: 1.42, 2.17) times higher expected rate of number of missed days at work due to illness or injury, respectively, compared with people with zero CMRFs. 
Table I Prevalence of CMRFs by number of risk factors (MEPS 2010-2012)

\begin{tabular}{|c|c|c|c|c|c|c|}
\hline \multirow[b]{2}{*}{ Population characteristics } & \multirow[b]{2}{*}{$\begin{array}{l}\text { Average MEPS } \\
\text { population }\end{array}$} & \multicolumn{4}{|c|}{ Prevalence of CMRFs ${ }^{a}$} & \multirow[b]{2}{*}{$\begin{array}{l}\text { Three or four } \\
\text { risk factors }\end{array}$} \\
\hline & & $\begin{array}{l}\text { Any risk } \\
\text { factor }\end{array}$ & $\begin{array}{l}\text { Zero risk } \\
\text { factors }\end{array}$ & $\begin{array}{l}\text { One risk } \\
\text { factor }\end{array}$ & $\begin{array}{l}\text { Two risk } \\
\text { factors }\end{array}$ & \\
\hline Sample size $(n)$ & - & 43,037 & 30,849 & 29,647 & 9,392 & 3,998 \\
\hline \multicolumn{7}{|l|}{$\operatorname{Sex}(\%)$} \\
\hline Male & 48.26 & 50.93 & 45.38 & 50.93 & 50.19 & 52.67 \\
\hline Female & 51.74 & 49.07 & 54.62 & 49.07 & 49.81 & 47.33 \\
\hline \multicolumn{7}{|l|}{ Age category, years (\%) } \\
\hline $18-34$ & 30.24 & 20.39 & 44.13 & 28.63 & 4.32 & 1.70 \\
\hline $35-49$ & 25.59 & 25.67 & 25.94 & 29.61 & 19.27 & $|3.7|$ \\
\hline $50-64$ & 25.86 & 29.91 & 20.66 & 24.83 & 39.68 & 41.75 \\
\hline $65-79$ & 13.25 & 17.87 & 6.67 & 11.73 & 28.11 & 35.90 \\
\hline $80+$ & 5.06 & 6.15 & 2.59 & 5.19 & 8.63 & 6.93 \\
\hline \multicolumn{7}{|l|}{ Race (\%) } \\
\hline White & 80.67 & 80.59 & 80.94 & 81.19 & 79.59 & 78.75 \\
\hline Black & 11.80 & 13.70 & 9.02 & 12.84 & 15.43 & 15.53 \\
\hline American Indian & 0.74 & 0.83 & 0.64 & 0.85 & 0.78 & 0.82 \\
\hline Other & 6.79 & 4.88 & 9.41 & 5.12 & 4.21 & 4.90 \\
\hline \multicolumn{7}{|l|}{ Ethnicity (\%) } \\
\hline Hispanic & $|4.6|$ & $15.0 \mid$ & 13.87 & 17.07 & 10.05 & 12.53 \\
\hline Non-Hispanic & 85.39 & 84.99 & 86.13 & 82.93 & 89.95 & 87.47 \\
\hline \multicolumn{7}{|l|}{ Region (\%) } \\
\hline Northeast & 18.30 & 17.52 & 18.99 & 17.62 & 17.42 & 17.06 \\
\hline Midwest & 21.51 & 22.17 & 20.84 & 21.66 & 23.12 & 23.47 \\
\hline South & 37.00 & 38.96 & 34.47 & 38.02 & 40.34 & 42.18 \\
\hline West & 23.19 & 21.35 & 25.70 & 22.71 & 19.12 & 17.30 \\
\hline \multicolumn{7}{|l|}{ Highest degree completed (\%) } \\
\hline No degree & $|4.7|$ & 14.39 & 14.47 & 13.43 & 15.08 & 19.37 \\
\hline Less than bachelors & 56.60 & 60.17 & 51.89 & 59.81 & 60.62 & 61.53 \\
\hline Bachelors & 18.69 & 16.50 & 21.99 & 17.46 & 15.69 & $1 \mathrm{I} .87$ \\
\hline Masters, PhD & 10.00 & 8.94 & 11.65 & 9.30 & 8.62 & 7.23 \\
\hline \multicolumn{7}{|l|}{ Family income category ${ }^{\mathrm{b}}(\%)$} \\
\hline Poor & 12.83 & 12.73 & 12.41 & 13.04 & 10.97 & 14.74 \\
\hline Near poor & 4.54 & 4.87 & 3.86 & 4.74 & 5.00 & 5.52 \\
\hline Low income & 13.67 & $|4.5|$ & 12.33 & 14.42 & 14.66 & 14.76 \\
\hline Middle income & 30.21 & 30.77 & 29.68 & 30.58 & 31.15 & 31.22 \\
\hline High income & 38.75 & 37.12 & 41.72 & 37.22 & 38.23 & 33.77 \\
\hline \multicolumn{7}{|l|}{ Insurance status (\%) } \\
\hline No insurance during year & 15.04 & 13.05 & 17.30 & 16.07 & 7.10 & 6.32 \\
\hline Private only during year & 53.87 & 49.37 & 61.61 & 54.06 & 43.11 & 31.94 \\
\hline Public only during year & 19.77 & 23.16 & 14.26 & 18.98 & 29.57 & 36.80 \\
\hline Public and private during year & 11.32 & 14.42 & 6.83 & 10.90 & 20.22 & 24.93 \\
\hline Age, years (mean) & 46.84 & 51.16 & 40.41 & 46.75 & 59.46 & 61.89 \\
\hline BMI, $\mathrm{kg} / \mathrm{m}^{2}$ (mean) & 27.75 & 31.19 & 23.09 & 30.43 & 32.10 & 34.32 \\
\hline Number of chronic conditions (mean) & 1.94 & 2.63 & 0.98 & 1.74 & 3.98 & 5.59 \\
\hline Number of chronic conditions excluding CMRFs ${ }^{c}$ (mean) & 1.35 & 1.69 & 0.87 & 1.29 & 2.33 & 2.95 \\
\hline
\end{tabular}

Notes: aCMRFs: abdominal obesity (BMI $\geq 27 \mathrm{~kg} / \mathrm{m}^{2}$ ), elevated blood pressure (at least one ICD-9 code for hypertension - $40 \mathrm{l} . \mathrm{x}$ ), elevated triglycerides (having at least one prescription), elevated glucose (at least one ICD-9 code for diabetes [250.xx] and at least one prescription for an antihyperglycemic medication), hyperuricemia (at least one ICD-9 code for gout [274.x] and at least one prescription). ${ }^{b}$ Family income poverty levels: poor, income less than or equal to poverty line; near poor, income over the poverty line through $125 \%$ of poverty line; low income, income over $125 \%$ through $200 \%$ of poverty line; middle income, income over $200 \%$ through $400 \%$ of poverty line; high income, income over $400 \%$ of poverty line. 'Number of chronic conditions excluding ICD-9 codes: 250, 272, 274, 278, and 40I.

Abbreviations: CMRFs, cardiometabolic risk factors; BMI, body mass index; ICD-9, International Classification of Diseases, ninth revision; MEPS, Medical Expenditure Panel Survey. 
Table 2 Prevalence of CMRFs by type of risk factor (MEPS 2010-2012)

\begin{tabular}{|c|c|c|c|c|c|}
\hline \multirow[t]{2}{*}{ Population characteristics } & \multirow[b]{2}{*}{$\begin{array}{l}\text { Average MEPS } \\
\text { population }\end{array}$} & \multicolumn{3}{|c|}{ Prevalence of CMRFs ${ }^{a}$} & \multirow[b]{2}{*}{$\begin{array}{l}\text { Elevated } \\
\text { glucose }\end{array}$} \\
\hline & & $\begin{array}{l}\text { Abdominal } \\
\text { obesity }\end{array}$ & $\begin{array}{l}\text { Elevated blood } \\
\text { pressure }\end{array}$ & $\begin{array}{l}\text { Elevated } \\
\text { triglycerides }\end{array}$ & \\
\hline Sample size $(n)$ & - & 36,638 & 16,830 & $\mathrm{I}, 756$ & 6,566 \\
\hline \multicolumn{6}{|l|}{$\operatorname{Sex}(\%)$} \\
\hline Male & 48.26 & 51.95 & 47.77 & 61.15 & 50.22 \\
\hline Female & 51.74 & 48.05 & 52.23 & 38.85 & 49.78 \\
\hline \multicolumn{6}{|l|}{ Age category, years (\%) } \\
\hline $18-34$ & 30.24 & 23.90 & 2.70 & 2.53 & 3.78 \\
\hline $35-49$ & 25.59 & 28.68 & $|4.2|$ & 16.60 & 15.92 \\
\hline $50-64$ & 25.86 & 29.74 & 37.28 & 39.20 & 38.68 \\
\hline $65-79$ & 13.25 & 14.46 & 32.43 & 34.96 & 32.82 \\
\hline $80+$ & 5.06 & 3.22 & 13.38 & 6.70 & 8.80 \\
\hline \multicolumn{6}{|l|}{ Race (\%) } \\
\hline White & 80.67 & 80.60 & 79.76 & 89.67 & 76.69 \\
\hline Black & 11.80 & 14.36 & 14.36 & 3.87 & 15.94 \\
\hline American Indian & 0.74 & 0.90 & 0.64 & 0.54 & 0.85 \\
\hline Other & 6.79 & 4.14 & 5.23 & 5.93 & 6.52 \\
\hline \multicolumn{6}{|l|}{ Ethnicity (\%) } \\
\hline Hispanic & $|4.6|$ & 16.38 & 8.81 & 9.64 & 14.75 \\
\hline Non-Hispanic & 85.39 & 83.62 & 91.19 & 90.36 & 85.25 \\
\hline \multicolumn{6}{|l|}{ Region (\%) } \\
\hline Northeast & 18.30 & 17.14 & 17.93 & 17.79 & 17.03 \\
\hline Midwest & 21.51 & 22.56 & 22.76 & 22.03 & 22.55 \\
\hline South & 37.00 & 38.79 & 40.52 & 41.13 & 41.19 \\
\hline West & 23.19 & 21.50 & 18.79 & 19.05 & 19.24 \\
\hline \multicolumn{6}{|l|}{ Highest degree completed (\%) } \\
\hline No degree & $|4.7|$ & $14.1 \mid$ & 15.73 & 15.67 & 20.58 \\
\hline Less than bachelors & 56.60 & 60.84 & 59.51 & 58.24 & 61.05 \\
\hline Bachelors & 18.69 & 16.40 & 15.56 & 15.97 & 11.92 \\
\hline Masters, PhD & 10.00 & 8.65 & 9.20 & 10.12 & 6.45 \\
\hline \multicolumn{6}{|l|}{ Family income category ${ }^{\mathrm{b}}(\%)$} \\
\hline Poor & 12.83 & 13.17 & 11.58 & 12.01 & 14.38 \\
\hline Near poor & 4.54 & 4.82 & 5.28 & 4.08 & 5.86 \\
\hline Low income & 13.67 & 14.36 & 14.78 & 12.62 & 16.39 \\
\hline Middle income & 30.21 & 31.17 & 30.23 & 29.52 & 31.72 \\
\hline High income & 38.75 & 36.48 & 38.13 & 41.78 & 31.65 \\
\hline \multicolumn{6}{|l|}{ Insurance status (\%) } \\
\hline No insurance during year & 15.04 & 14.67 & 5.88 & 4.23 & 7.70 \\
\hline Private only during year & 53.87 & 53.20 & 36.16 & 37.30 & 32.45 \\
\hline Public only during year & 19.77 & 20.73 & 33.23 & 31.41 & 37.14 \\
\hline Public and private during year & $11.32 \%$ & 11.39 & 24.73 & 27.05 & 22.71 \\
\hline Age, years (mean) & 46.84 & 48.27 & 62.72 & 61.06 & 60.88 \\
\hline BMI, kg/m² (mean) & 27.75 & 32.66 & 30.07 & 30.71 & 32.06 \\
\hline Number of chronic conditions (mean) & 1.94 & 2.34 & 4.39 & 5.10 & 5.04 \\
\hline Number of chronic conditions excluding $\mathrm{CMRFs}^{c}$ (mean) & 1.35 & 1.54 & 2.54 & 3.05 & 2.61 \\
\hline
\end{tabular}

Notes: aCMRFs: abdominal obesity $\left(\mathrm{BMI} \geq 27 \mathrm{~kg} / \mathrm{m}^{2}\right.$ ), elevated blood pressure (at least one ICD-9 code for hypertension - $40 \mathrm{I} . \mathrm{x}$ ), elevated triglycerides (having at least one prescription), elevated glucose (at least one ICD-9 code for diabetes [250.xx] and at least one prescription for an antihyperglycemic medication), hyperuricemia (at least one ICD-9 code for gout [274.x] and at least one prescription). ${ }^{b}$ Family income poverty levels: poor, income less than or equal to poverty line; near poor, income over the poverty line through 125\% of poverty line; low income, income over 125\% through $200 \%$ of poverty line; middle income, income over $200 \%$ through $400 \%$ of poverty line; high income, income over $400 \%$ of poverty line. 'Number of chronic conditions excluding ICD-9 codes: 250, 272, 274, 278, and 40I.

Abbreviations: CMRFs, cardiometabolic risk factors; BMI, body mass index; ICD-9, International Classification of Diseases, ninth revision; MEPS, Medical Expenditure Panel Survey. 
Table 3 Annual utilization and expenditure estimates by number of CMRFs

\begin{tabular}{|c|c|c|c|c|c|c|c|c|c|}
\hline \multirow[t]{3}{*}{ Variables } & \multicolumn{9}{|c|}{ Number of CMRFs } \\
\hline & \multirow{2}{*}{$\begin{array}{l}\text { Average } \\
\text { MEPS } \\
\text { population }^{\mathrm{a}}\end{array}$} & \multicolumn{2}{|c|}{$\begin{array}{l}\text { Zero risk factors } \\
(n=30,849)\end{array}$} & \multicolumn{2}{|c|}{$\begin{array}{l}\text { One risk factor } \\
(n=29,647)\end{array}$} & \multicolumn{2}{|c|}{$\begin{array}{l}\text { Two risk factors } \\
(n=9,392)\end{array}$} & \multicolumn{2}{|c|}{$\begin{array}{l}\text { Three or four risk } \\
\text { factors }(n=3,998)\end{array}$} \\
\hline & & Unadjusted & Adjusted & Unadjusted $^{a}$ & Adjusted & Unadjusted $^{\mathbf{a}}$ & Adjusted & Unadjusted $^{a}$ & Adjusted \\
\hline \multicolumn{10}{|l|}{ Utilization $^{\mathrm{b}}$} \\
\hline $\begin{array}{l}\text { Number of } \\
\text { outpatient visits }\end{array}$ & 8.11 & 5.57 & $\begin{array}{l}\text { Reference } \\
\text { group }\end{array}$ & 7.55 & $\begin{array}{l}1.08 \\
(1.02,1.15)\end{array}$ & 12.84 & $\begin{array}{l}1.26 \\
(1.17,1.35)\end{array}$ & 17.59 & $\begin{array}{l}1.55 \\
(1.42,1.70)\end{array}$ \\
\hline $\begin{array}{l}\text { Number of } \\
\text { emergency room } \\
\text { visits }\end{array}$ & 0.19 & 0.14 & $\begin{array}{l}\text { Reference } \\
\text { group }\end{array}$ & 0.19 & $\begin{array}{l}1.15 \\
(1.06,1.24)\end{array}$ & 0.28 & $\begin{array}{l}1.37 \\
(1.25,1.51)\end{array}$ & 0.31 & $\begin{array}{l}1.39 \\
(1.22,1.57)\end{array}$ \\
\hline $\begin{array}{l}\text { Number of } \\
\text { inpatient visits }\end{array}$ & 0.11 & 0.07 & $\begin{array}{l}\text { Reference } \\
\text { group }\end{array}$ & 0.10 & $\begin{array}{l}1.14 \\
(1.04,1.25)\end{array}$ & 0.19 & $\begin{array}{l}1.50 \\
(1.22,1.69)\end{array}$ & 0.26 & $\begin{array}{l}1.77 \\
(1.51,2.08)\end{array}$ \\
\hline $\begin{array}{l}\text { Inpatient length } \\
\text { of stay (days) }\end{array}$ & 0.59 & 0.28 & $\begin{array}{l}\text { Reference } \\
\text { group }\end{array}$ & 0.46 & $\begin{array}{l}1.16 \\
(0.98,1.37)\end{array}$ & 0.97 & $\begin{array}{l}1.50 \\
(1.22,1.83)\end{array}$ & 1.44 & $\begin{array}{l}2.43 \\
(1.86,3.16)\end{array}$ \\
\hline $\begin{array}{l}\text { Number of } \\
\text { medications } \\
\text { including refills }\end{array}$ & 13.04 & 5.57 & $\begin{array}{l}\text { Reference } \\
\text { group }\end{array}$ & 10.77 & $\begin{array}{l}1.42 \\
(1.36,1.48)\end{array}$ & 28.49 & $\begin{array}{l}3.36 \\
(3.18,3.54)\end{array}$ & 49.76 & $\begin{array}{l}5.29 \\
(4.19,5.72)\end{array}$ \\
\hline $\begin{array}{l}\text { Health care } \\
\text { expenditure } \\
(2014 \text { US dollars) }\end{array}$ & $\$ 5,465$ & $\$ 3,523$ & $\begin{array}{l}\text { Reference } \\
\text { group }\end{array}$ & $\$ 4,763$ & $\begin{array}{l}\$ 417 \\
(\$ 70, \$ 763)\end{array}$ & $\$ 9,281$ & $\begin{array}{l}\$ 2,326 \\
(\$ 1,864, \\
\$ 2,788)\end{array}$ & $\$ 13,304$ & $\begin{array}{l}\$ 4,117 \\
(\$ 3,428, \\
\$ 4,807)\end{array}$ \\
\hline
\end{tabular}

Notes: anadjusted estimates are mean. 'Incidence rate ratio and $95 \%$ confidence intervals from negative binomial regression models after adjusting for age, sex, race, ethnicity, region, education, number of other chronic conditions, family income, insurance status, eg, after adjusting for all measured variables, those with three or four risk factors on average had a 1.55 higher expected rate of outpatient visits vs those with zero risk factors. 'Adjusted increments annual expenditures and $95 \%$ confidence intervals from generalized linear models with log link and gamma family distribution after adjusting for age, sex, race, ethnicity, region, education, number of other chronic conditions, family income, and insurance status.

Abbreviations: CMRFs, cardiometabolic risk factors; MEPS, Medical Expenditure Panel Survey.

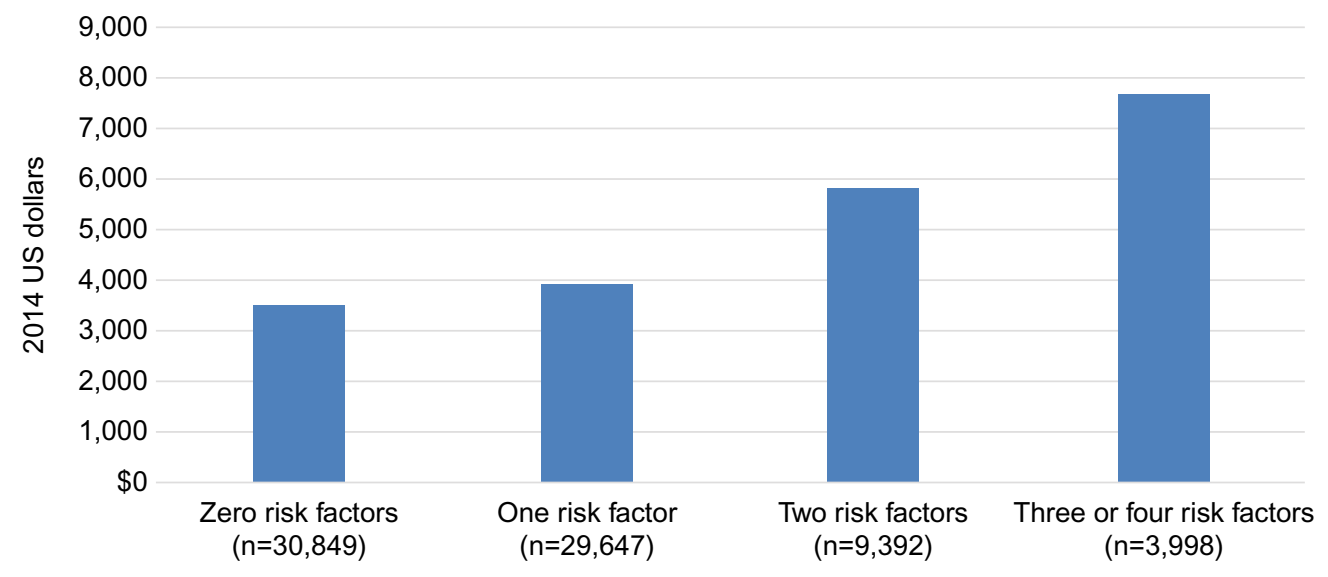

Figure I Adjusted annual health care expenditures by number of CMRFs for MEPS respondents from 2010 to 2012.

Note: Adjusted expenditures calculated from generalized linear models with log link and gamma family distribution after adjusting for clinical and demographic characteristics. Abbreviations: CMRFs, cardiometabolic risk factors; MEPS, Medical Expenditure Panel Survey.

The results of the sensitivity analyses were similar to the overall results presented. When three or four risk factors were split into separate categories, outcome measures were not significantly different from each other; therefore, we kept the three or four CMRF groups merged together for the main findings.

\section{Discussion}

The results of this study suggest the economic burden associated with CMRFs increases as the number of CMRFs increase. This association was independent of the burden associated with $\mathrm{CV}$ disease. Our findings were consistent across utilization, expenditure, and productivity outcomes.

Our findings are similar to previous research that found a significant economic burden of CMRFs independent of the cost of CV disease. Sullivan et al estimated the medical cost of CMRF clusters in the US using MEPS data from 2000 and 2002. ${ }^{10}$ The authors grouped four risk factors (ie, BMI $\geq 25 \mathrm{~kg} / \mathrm{m}^{2}$, diabetes, hypertension, and hyperlipidemia) to estimate overall utilization and expenditure differences for 
Table 4 Employment and absenteeism estimates by number of CMRFs

\begin{tabular}{|c|c|c|c|c|c|c|c|c|c|}
\hline \multirow[t]{3}{*}{ Variables } & \multicolumn{9}{|c|}{ Number of CMRFs } \\
\hline & \multirow{2}{*}{$\begin{array}{l}\text { Average } \\
\text { MEPS } \\
\text { population }^{\mathrm{a}}\end{array}$} & \multicolumn{2}{|c|}{$\begin{array}{l}\text { Zero risk factors } \\
(n=30,849)\end{array}$} & \multicolumn{2}{|c|}{$\begin{array}{l}\text { One risk factor } \\
(n=29,647)\end{array}$} & \multicolumn{2}{|c|}{$\begin{array}{l}\text { Two risk factors } \\
(n=9,392)\end{array}$} & \multicolumn{2}{|c|}{$\begin{array}{l}\text { Three or four risk } \\
\text { factors }(n=3,998)\end{array}$} \\
\hline & & Unadjusted & Adjusted & Unadjusted & Adjusted & Unadjusted & Adjusted & Unadjusted & Adjusted \\
\hline $\begin{array}{l}\text { Not employed } \\
\text { (\% on annual } \\
\text { basis) }{ }^{\mathrm{a}}\end{array}$ & $20.7 \%$ & $19.6 \%$ & $\begin{array}{l}\text { Reference } \\
\text { group }\end{array}$ & $18.3 \%$ & $\begin{array}{l}0.81 \\
(0.76,0.87)\end{array}$ & $26.1 \%$ & $\begin{array}{l}0.96 \\
(0.87,1.07)\end{array}$ & $39.6 \%$ & $\begin{array}{l}1.38 \\
(1.17,1.63)\end{array}$ \\
\hline $\begin{array}{l}\text { Annual number } \\
\text { of days missed } \\
\text { at work due to } \\
\text { illness or injury }\end{array}$ & 3.56 & 2.82 & $\begin{array}{l}\text { Reference } \\
\text { group }\end{array}$ & 3.69 & $\begin{array}{l}1.21 \\
(1.09,1.34)\end{array}$ & 5.71 & $\begin{array}{l}1.55 \\
(1.31,1.82)\end{array}$ & 6.65 & $\begin{array}{l}1.75 \\
(1.42,2.17)\end{array}$ \\
\hline
\end{tabular}

those with and without CMRFs, and to compare attributable CMRF expenditures by insurance type. The authors found adjusted annual total health care expenditures of $\$ 5,477$ in 2005 US dollars attributable to CMRFs when including BMI $\geq 25 \mathrm{~kg} / \mathrm{m}^{2}$, and at least two CMRFs out of diabetes, hypertension, and hyperlipidemia. Using recently updated MEPS data, our study, when analyzed differently, found a similarly large difference of US\$4,117 in adjusted annual health care expenditures for those with at least three or four CMRFs vs those with zero CMRFs.

Sullivan et al published a follow-up study using the same data (MEPS 2000 and 2002) and assumptions (BMI $\geq 25 \mathrm{~kg} / \mathrm{m}^{2}$ ) but focused solely on lost productivity-related outcomes. ${ }^{11}$ The annual number of missed work-days due to illness or injury attributable to CMRFs when including BMI $\geq 25 \mathrm{~kg} / \mathrm{m}^{2}$, and at least two CMRFs out of diabetes, hypertension, and hyperlipidemia, increased by a factor of 1.79 when compared with those without CMRFs. These results are similar to our findings as individuals with three or four risk factors had an increase in missed work-days due to illness and injury by a factor of 1.75 . But our study differs from these two studies by Sullivan et al in that we used the most recently updated MEPS-HC files. We categorized and estimated CMRFs by the number of CMRFs as opposed to a population with and without any CMRFs, and we used a different BMI stratification (BMI $\geq 27 \mathrm{~kg} / \mathrm{m}^{2}$ ).

Our results are also similar to previous studies that have analyzed medical costs by the number of CMRFs in combination with specific conditions such as coronary artery disease (CAD), diabetes, and obesity. Using a claims data set, Tamariz et al analyzed health care paid claims by CAD and by the number of CMRFs from one to four. ${ }^{15}$ Their popula- tion included individuals with at least one of the following conditions: diabetes, hypertension, abnormal lipid panel, and obesity. Health care expenditures were estimated with and without $\mathrm{CAD}$ and by the number of CMRFs. Although not directly comparable to our results, the observed trend of increasing expenditures associated with CMRFs is consistent with our findings. For example, adjusted health care expenditures in 2003-2004 US dollars were $\$ 4,117, \$ 4,968$, $\$ 6,173$, and $\$ 9,276$ for one, two, three, and four CMRFs, respectively. Adjusted health care expenditure estimates more than doubled when CAD was included.

Sullivan et al conducted a CMRF-related study using MEPS data but estimated health care expenditures and lost productivity attributable to CMRFs and comorbid obesity (ie, BMI $\geq 30 \mathrm{~kg} / \mathrm{m}^{2}$ ). ${ }^{16}$ Their findings suggested significantly higher medical expenditures and lost productivity for normal weight individuals with diabetes, dyslipidemia, or hypertension as compared with those without those conditions. Obesity significantly increased those expenditures and lostproductivity estimates. For example, obese individuals with three risk factors had an increase of nearly \$10,000 2007 US dollars in annual total adjusted health care expenditures as compared to normal weight individuals with zero risk factors.

Boudreau et al conducted a CMRF study in a managed care population, estimating health care costs with and without CMRFs, and by the number of CMRFs stratified by diabetes diagnosis. ${ }^{12} \mathrm{CMRF}$ included hypertension, weight risk (BMI $>27 \mathrm{~kg} / \mathrm{m}^{2}$ ), high triglycerides, low high-density lipoprotein, and diabetes. The most expensive group of CMRFs included diabetes, dyslipidemia, and hypertension that had total annual costs of $\$ 9,650$ in 2005 US dollars. A $24 \%$ increase in total annual health care costs per additional 
risk factor was demonstrated with similar trends observed when stratified by diabetes status. Although the percentage increase and stratification of diabetes status were not similar to our study, these results are consistent with our findings that health care expenditures significantly increase for people with at least two to four CMRFs as compared with people without CMRFs. Boudreau et al suggested focusing on specific metabolic syndrome risk factor combinations for health care resource planning. Our study differs from these studies as our primary focus is on the number of CMRFs, regardless of a specific comorbid condition such as obesity, diabetes, or CAD. Moreover, we used the most recently available nationally representative survey data from MEPS and present lost-productivity outcomes as an additional source of the economic burden attributable to CMRFs.

\section{Limitations}

Our study has important limitations that impact the interpretation of the results. This study was cross-sectional where CMRF stratification and economic burden outcomes were measured contemporaneously. This may result in higher than actual health care expenditure estimates considering that CMRF definitions were based on utilization of health care resources such as prescription medications. We did not estimate health care resource utilization and expenditures when CMRFs were linked with CV disease events, or other conditions such as diabetes. There has been previous research suggesting that composite measures of the metabolic syndrome (ie, all CMRFs included) are not significant predictors of health care resource utilization. ${ }^{12,19}$ Rather, some have suggested individual CMRFs such as abdominal obesity, comorbid conditions such as diabetes, or specific combinations of CMRFs and comorbid conditions (eg, hypertension and diabetes) are the drivers of high utilization. Our study findings suggest that regardless of specific CMRFs, significant health care resources are consumed when patients have a combination of CMRFs as compared with patients with no CMRFs.

CMRF definitions were based on self-reported conditions from MEPS. This may represent an underreporting of included conditions ${ }^{20}$ and thus an underestimate of the economic burden. However, prevalence estimates reported here are similar, in some cases lower or higher, to other studies relying on recent self-reported data. ${ }^{8,21}$ For example, as compared with data using National Health and Nutrition Examination Survey (NHANES), we found a higher prevalence estimate of abdominal obesity, yet slightly lower prevalence of elevated blood pressure and elevated glucose. Some of these differences are attributable to dif- ferent cutoff points for CMRFs. Overweight individuals specifically may underestimate their weight and overestimate their height. ${ }^{22,23}$ Furthermore, MEPS does not contain information on undiagnosed conditions such as NHANES does, which may also lead to underestimated prevalence of these CMRFs.

\section{Conclusion}

This study contributes to the body of literature suggesting as the number of CMRFs increase, regardless of specific underlying conditions, the economic burden increases for both direct health care expenditures and lost productivity. Prevalence of elevated glucose and elevated waist circumference are still on the rise. These results underscore the value of providing incentives to reduce the burden of these CMRFs from an economic and productivity standpoint. More attention should focus on treating the underlying metabolic syndrome instead of focusing on one condition alone.

\section{Acknowledgment}

This study was supported by AstraZeneca (London, UK) on an unrestricted grant.

\section{Disclosure}

RBM, VG, KVN, and JJS received research support from AstraZeneca to conduct this study. TO and JJ Sheehan are employees of AstraZeneca. The authors report no other conflicts of interest in this work.

\section{References}

1. Grundy SM, Cleeman JI, Daniels SR, et al. Diagnosis and management of the metabolic syndrome: an American Heart Association/National Heart, Lung, and Blood Institute Scientific Statement. Circulation. 2005;112(17):2735-2752.

2. Flint AJ, Hu FB, Glynn RJ, et al. Excess weight and the risk of incident coronary heart disease among men and women. Obesity (Silver Spring). 2010;18(2):377-383.

3. Ford ES. Risks for all-cause mortality, cardiovascular disease, and diabetes associated with the metabolic syndrome: a summary of the evidence. Diabetes Care. 2005;28(7):1769-1778.

4. Lakka HM, Laaksonen DE, Lakka TA, et al. The metabolic syndrome and total and cardiovascular disease mortality in middle-aged men. JAMA. 2002;288(21):2709-2716.

5. Malik S, Wong ND, Franklin SS, et al. Impact of the metabolic syndrome on mortality from coronary heart disease, cardiovascular disease, and all causes in United States adults. Circulation. 2004;110(10):1245-1250.

6. Wang J, Ruotsalainen S, Moilanen L, Lepisto P, Laakso M, Kuusisto J. The metabolic syndrome predicts cardiovascular mortality: a 13-year follow-up study in elderly non-diabetic Finns. Eur Heart J. 2007;28(7):857-864.

7. Mozaffarian D, Kamineni A, Prineas RJ, Siscovick DS. Metabolic syndrome and mortality in older adults: the Cardiovascular Health Study. Arch Int Med. 2008;168(9):969-978.

8. Mozaffarian D, Benjamin EJ, Go AS, et al. Heart disease and stroke statistics - 2015 update: a report from the American Heart Association. Circulation. 2015;131(24):e535. 
9. Kannan H, Thompson S, Bolge SC. Economic and humanistic outcomes associated with comorbid type-2 diabetes, high cholesterol, and hypertension among individuals who are overweight or obese. J Occup Environ Med. 2008;50(5):542-549.

10. Sullivan PW, Ghushchyan V, Wyatt HR, Hill JO. The medical cost of cardiometabolic risk factor clusters in the United States. Obesity (Silver Spring). 2007;15(12):3150-3158.

11. Sullivan PW, Ghushchyan V, Wyatt HR, Wu EQ, Hill JO. Productivity costs associated with cardiometabolic risk factor clusters in the United States. Value Health. 2007;10(6):443-450.

12. Boudreau DM, Malone DC, Raebel MA, et al. Health care utilization and costs by metabolic syndrome risk factors. Metab Syndr Relat Disord. 2009;7(4):305-314.

13. Weycker D, Nichols GA, O'Keeffe-Rosetti M, et al. Risk-factor clustering and cardiovascular disease risk in hypertensive patients. Am J Hypertens. 2007;20(6):599-607.

14. Brixner DI, Bron M, Bellows BK, et al. Evaluation of cardiovascular risk factors, events, and costs across four BMI categories. Obesity (Silver Spring). 2013;21(6):1284-1292.

15. Tamariz L, Palacio A, Yang Y, Parris D, Ben-Joseph R, Florez H. Health economics perspective of the components of the cardiometabolic syndrome. J Clin Hypertens (Greenwich, Conn.). 2010;12(7):549-555.
16. Sullivan PW, Ghushchyan V, Ben-Joseph RH. The effect of obesity and cardiometabolic risk factors on expenditures and productivity in the United States. Obesity (Silver Spring). 2008;16(9):2155-2162.

17. Agency for Healthcare Research and Quality. Medical Expenditure Panel Survey. 2013. Available from: http://meps.ahrq.gov/mepsweb/. Accessed July 1, 2015.

18. Orzano AJ, Scott JG. Diagnosis and treatment of obesity in adults: an applied evidence-based review. J Am Board Fam Pract. 2004;17(5):359-369.

19. Curtis LH, Hammill BG, Bethel MA, Anstrom KJ, Gottdiener JS, Schulman KA. Costs of the metabolic syndrome in elderly individuals: findings from the Cardiovascular Health Study. Diabetes Care. 2007;30(10):2553-2558.

20. Evaluation of National Health Interview Survey diagnostic reporting. Vital Health Stat 2. 1994(120):1-116.

21. Yang L, Colditz GA. Prevalence of overweight and obesity in the United States, 2007-2012. JAMA Intern Med. 2015;175(8):1412-1413.

22. Rowland ML. Self-reported weight and height. Am J Clin Nutr. 1990;52(6):1125-1133.

23. Zarychta K, Mullan B, Luszczynska A. It doesn't matter what they say, it matters how they behave: parental influences and changes in body mass among overweight and obese adolescents. Appetite. 2015;96:47-55.
Diabetes, Metabolic Syndrome and Obesity: Targets and Therapy is an international, peer-reviewed open-access journal committed to the rapid publication of the latest laboratory and clinical findings in the fields of diabetes, metabolic syndrome and obesity research. Original research, review, case reports, hypothesis formation, expert opinion and commentaries are all considered for publication. The manuscript management system is completely online and includes a very quick and fair peer-review system, which is all easy to use. Visit http://www.dovepress.com/testimonials.php to read real quotes from published authors.

Submit your manuscript here: https://www.dovepress.com/diabetes-metabolic-syndrome-and-obesity-targets-and-therapy-journal 